\title{
High and Low Stimulus-Driven Conflict Engage Segregated Brain Networks, Not Quantitatively Different Resources
}

\author{
Leila Chouiter · Sebastian Dieguez $\cdot$ \\ Jean-Marie Annoni $\cdot$ Lucas Spierer
}

Received: 11 March 2013/Accepted: 12 June 2013/Published online: 29 June 2013

(c) Springer Science+Business Media New York 2013

\begin{abstract}
Task-irrelevant information is constantly present in our environment and may interfere with the processing of the information necessary to achieve goaldirected behavior. While task goals determine which information must be suppressed, the demand for inhibitory control depends on the strength of the interference induced by incoming, task-irrelevant information. Whether the same or distinct inhibitory processes are engaged to suppress various degrees of interference from task-irrelevant information remains largely unresolved. We investigated this question by manipulating the strength of the conflict induced by automatic word reading in a classical color Stroop task. High conflict was induced by presenting words in participant's native language and low conflict by presenting words in a less familiar language. Behavioral performance and electrical neuroimaging analyses of eventrelated potentials to the words were analyzed following a two-by-two within-subject design with factors conflict strength (high; low) and color word/word ink congruency (congruent; incongruent). Behaviorally, we observed a significant conflict strength $\times$ congruency driven by a smaller Stroop effect in the low- than high conflict condition. Electrophysiologically, we observed a significant conflict strength $\times$ congruency interaction at the topographic level during the period of the N450 components,
\end{abstract}

Electronic supplementary material The online version of this article (doi:10.1007/s10548-013-0303-0) contains supplementary material, which is available to authorized users.

L. Chouiter $(\bowtie) \cdot$ S. Dieguez $\cdot$ J.-M. Annoni - L. Spierer Neurology Unit, Laboratory for Cognitive and Neurological Sciences, Medicine Department, Faculty of Sciences, University of Fribourg, PER 09, Chemin du Musée 5, 1700 Fribourg, Switzerland e-mail: leila.chouiter@unifr.ch indicative of the engagement of distinct configurations of brain networks. No such interaction was found at the level of response strength. Electrical sources analyses localized the topographic effect within the anterior cingulate cortex and basal ganglia, left middle frontal and occipital areas. We interpret our results in terms of qualitatively distinct executive mechanisms for reactive inhibitory control in conditions of high versus low stimulus-driven conflict.

Keywords Inhibitory control - EEG - Topography · Electrical source estimation

\section{Introduction}

During goal-directed behavior, task-irrelevant information may interfere with the information relevant to attain one's goals and ultimately hinder performance. "Dual pathway" models (Abrams et al. 1990; Ridderinkhof and van der Molen 1997) advance that task-relevant information is processed by top-down controlled pathways, which activate the response patterns matching with the task goals. Yet, the stimuli can also convey task-irrelevant information, which may activate automatic responses via bottomup processing pathways and interfere with the response pattern elicited by the top-down route. Because the inhibitory control mechanisms engaged to suppress irrelevant information take time, response speed in conflict tasks depends on the strength of the interfering activation of the bottom-up pathway. The nature of such interfering activations, however, is still poorly understood. Here we investigate the role of the strength of bottom-up interference with a robust within-subjects design.

Stroop color tasks, consisting in reporting the color of color words while ignoring the color named by the words, 
have been widely used to investigate the inhibitory control of task-irrelevant information (Stroop 1935). Because the semantic information on color conveyed by the automatic reading of the words interferes with the information from the color of the word, increases in response times (RTs) are typically observed when the color of the word is incongruent with the color named by the word, as compared with trials where the color of the word matches the color named by the word, (so-called 'Stroop effect; Logan 1980; MacLeod and Dunbar 1988; MacLeod 1991).

Converging functional neuroimaging evidence indicates that the anterior cingulate cortex (ACC), the dorsolateral prefrontal cortex (DLPFC) and parietal regions are sensitive to the conflict induced by task-irrelevant information at ca. $450 \mathrm{~ms}$ post-stimulus onset and involved in response selection (Khateb et al. 2000; Liotti et al. 2000; Botvinick et al. 2001; Tillman and Wiens 2011). Although triggered by the conflicting stimuli, inhibitory control mechanisms are mostly proactive; they are set up according to task instructions and bias information processing to facilitate the integration of task-relevant information over task-irrelevant inputs (Kastner and Ungerleider 2000; Miller and Cohen 2001; Corbetta and Shulman 2002; Braver et al. 2009; Morishima et al. 2009). Recent evidence, however, pointed out that reactive control mechanisms might also be necessary to dynamically adjust the levels of control depending on the strength of the interference from the task-irrelevant information. Such reactive inhibitory control mechanisms have been notably posited by Morishima et al. (2010), who showed that spontaneous fluctuations in the amount of conflict during a Stroop task modulated the engagement of the DLPFC and that interactions between the ACC and the DLPFC influenced the behavioral outcome in high-conflict trials. However, this study focused on the effect of endogenous sources of trial to trial variation in conflict strength, which could be assumed to be of lower amplitude and qualitatively different from modulations in conflict strength driven by external events. Whether and how distinct stimulus-driven reactive inhibitory control mechanisms are engaged in situations of high versus low levels of conflict from task-irrelevant information remains poorly understood.

Insight into this question comes from behavioral studies in which Stroop effects were contrasted between conditions where the interference from the task-irrelevant word meaning information was high, as induced by writing the color words in the native language of the participant, and conditions in which the interference was low, as induced by writing the word in a less familiar language. These studies showed that degree of familiarity of the language in which words were presented modulated Stroop effects, with smaller conflicts when the color words were written in an unfamiliar than in a familiar language (Grass 1984; Mohamed Zied et al. 2004; Sumiya and Healy 2004, 2008;
Braet et al. 2011; Aron 2011; though see Lee and Chan 2000). These studies parsimoniously interpreted such modulation in terms of the same inhibitory control process being engaged independently of strength of the Stroop conflict, but more strongly in high than low conflict situations. Yet, this assumption lacks direct empirical support; whether the inhibitory process engaged to control high versus low differs only quantitatively or involves distinct configurations of brain networks remains unclear. A recent functional neuroimaging Stroop study by Youn (2011) investigated the neural correlates of Korean and English Stroop tasks in native Korean speakers, who have received at least 6 years of English education after 7 years old. The author found that the basal ganglia, thalamus, ACC, right inferior frontal gyrus and the middle temporal gyrus were more activated in the incongruent Korean than in the incongruent English conditions. In contrast, the bilateral superior temporal, parahippocampal, left fusiform, medial frontal and precentral gyri, the caudate nucleus, cuneus right inferior parietal lobule, and the insula were more activated in the incongruent English than in the incongruent Korean condition. However, although this study speaks in favor of qualitatively distinct inhibitory control mechanisms depending on the conflict strength (as manipulated by presenting the words in the native (Korean) versus less familiar (English) language), it did not test directly an interaction between the degree of conflict and the factor of congruency (Nieuwenhuis and Donner 2011).

To resolve the spatio-temporal brain mechanisms underlying reactive inhibitory control processes in high versus low stimulus-driven conflict conditions, we applied electrical neuroimaging analyses to event-related potentials (ERPs) recorded while participants completed a classical color Stroop paradigm with stimuli inducing either a high (words written in the native language) or low (word written in a second, low-proficient language) conflict with the taskrelevant ink color information. To ensure that proactive control mechanisms did not differ between conditions and that our analysis revealed only differences in reactive, stimulus-driven mechanisms, the two conflict conditions were randomly intermixed within each block.

\section{Rationale and Hypotheses}

Differences in inhibitory processes engaged during the Stroop task were analyzed by applying time-wise, datadriven randomization statistics on the configuration (i.e. the topography) and the strength of the scalp-recorded electric field according to a 2 by 2 design with conflict strength (high; low) and color word/word ink congruency (congruent; incongruent) as within-subjects factors. As compared to the classical analyses of ERP components at the level of local electrodes, analyses of the modulation in the 
topography and in the strength of the voltage field allow to disentangle whether the measured effects follow from changes in the configuration of the underlying brain networks and/or in their response strength: A change in the topography of the electric field indeed necessarily follows from a modification in the configuration of the underlying intracranial generators (Lehmann and Skrandies 1980; Lehmann et al. 1987). In contrast, a modification in the strength of the electric field without concomitant topographic change can be interpreted as a modulation in the response strength between statistically indistinguishable brain networks (Koenig and Melie-Garcia 2010; Tzovara et al. 2011, 2012 for review). Topographic and global field power modulations can thus be respectively interpreted in terms of the engagement of qualitatively or quantitatively different brain networks across experimental conditions. To localize in the brain the sources of the effects measured at the scalp, statistical analyses of distributed electrical source estimations were conducted over periods of significant ERP modulations.

\section{Materials and Methods}

\section{Participants}

Twelve healthy right-handed volunteers participated in the study ( 2 women; laterality was assessed using the Edinburgh questionnaire by Oldfield 1971), aged 22-37 years (mean $\pm \mathrm{SD}, \quad 24.4 \pm 4.8$ years). All participants were native German speakers (German was thus used as the high conflict (HC) condition), who were in the process of learning French as a second language (low conflict (LC) condition). To ensure that presenting the words in French induced a weaker conflict than in the German condition, we selected participant that learned French after the age of seven and had a medium proficiency level (see the "Assessment of Language Proficiency" section). No participant had a history of neurological or psychiatric illness and all reported normal hearing. Each participant provided written, informed consent to participate in the study. All procedures were approved by the Ethics Committee of the University of Fribourg.

\section{Assessment of Language Proficiency}

Two questionnaires were used to assess the participant's second language proficiency. The tests were completed by participants prior to the experiment in order to determine whether his/her proficiency matched our inclusion criteria of medium proficiency. This criterion ensured that the high and low conflict conditions actually differed in the strength of the conflict induced by automatic reading during the Stroop task.
The Oxford University Placement Test was used to assess L2 level. This test consists of 50 multi-choices questions on grammar, vocabulary and conjugation (http:// www.lang.ox.ac.uk/courses/tst_placement_english.htm). The boston naming test (BNT) was conducted in both L1 and L2. This is a picture naming vocabulary test consisting of 34 items (Kaplan et al. 1983)

\section{Stimuli}

Stimuli were four French and four German color words (French/German (English) word: "noir"/"schwarz" (black), "vert"/"grün" (green), "jaune"/"gelb" (yellow), "blanc"/ "weiss" (white)) presented centrally on a grey background during $200 \mathrm{~ms}$. These colors were chosen because they enabled us to have between-language phonemic differences without between-languages differences in words' length. Each color name was presented according to two conditions: In the congruent condition (hereafter termed " $\mathrm{C}$ "), the ink color and the color word referred to the same color (e.g. "black" written in black) and in the incongruent condition ("I"), the ink color and the color word referred to different colors (e.g. "black" written in green).

\section{Procedure and Task}

Participants were seated in a comfortable armchair in front of a LCD display screen. They indicated the color of the ink using manual responses to avoid having to determine the language in which participants responded and to contaminate our effects with processes related to language production (for similar approach see e.g. Mead et al. 2002; Peterson et al. 2002; Liu et al. 2004; van Veen and Carter 2005; Britz and Michel 2010). Participants responded using their right hand during the whole experiment. They were instructed to respond with the index finger for the first button corresponding to the response "black", middle finger for the second button "yellow", the ring finger for the third button "green" and the little finger for the fourth button "white". The button-color mapping rule was kept constant across the whole session. Stimulus presentation and response recording were controlled by the E-prime 2.0 Pro software.

The participants underwent two different tasks. First, each participant completed a simple color discrimination block in which they were instructed to report, using the response box, the color named by four different words. This familiarization task served to train the participants to associate each color with a button with the aim of facilitating responses and of reducing the effects of learning during the main EEG task. In this first block, all words were written in pink to avoid any interference between the color of the ink and the color named by the words (the ink color and word pink were not used in the main EEG 
experiment). Twenty-four trials $(3$ trials $\times 4$ color words $\times 2$ languages) were presented in a single block to establish the mapping between the colors and the response buttons.

After learning the stimulus-response mapping rules, participants performed a classical bilingual color Stroop task (e.g. Lee and Chan 2000; Rosselli et al. 2002). They were instructed to report as fast as possible the color of the ink of the word irrespective of the color named by the word by pressing the corresponding button. All participants completed 10 blocks of 96 trials. Within each block, the color words were presented either in the $\mathrm{HC}$ or in the LC condition. The $\mathrm{HC}$ and $\mathrm{LC}$ conditions were intermixed and presented in a randomized order to avoid any difference in proactive inhibitory mechanisms (see "Introduction" and "Discussion" sections). In each block, there were 24 congruent HC trials (e.g. "grün" written with a green ink), 24 incongruent $\mathrm{HC}$ trials (e.g. "grün" in yellow), 24 congruent LC trials (e.g. "vert" in green) and 24 incongruent LC trials (e.g. "vert" in yellow). The number of each color word - ink color association was balanced across conditions within each block. Each trial started with a fixation cross lasting for a random inter-stimulus-interval (ISI) chosen between 1,000 and 2,000 ms (Fig. 1). Then, a target word appeared on the screen during $200 \mathrm{~ms}$ from the onset of the word, participants had a maximum of 2,000 $\mathrm{ms}$ to respond. A feedback (200 ms duration) informed the participant about his/her performance (correct, incorrect or too late) $700 \mathrm{~ms}$ after his/her response.

\section{Behavioral Analyses}

We indexed behavioral performance in the Stroop task by averaging RTs for each of the four conditions separately (C/HC; I/HC; C/LC; and I/LC). RTs of incorrect trials and extreme RTs higher or lower than the individual participant mean $\mathrm{RT} \pm 2 \mathrm{SD}$ were excluded prior to the behavioral analyses. The behavioral data were then submitted to a $2 \times 2$ repeated measure ANOVA with conflict strength and congruency as within-subject factors.

\section{EEG Analyses}

\section{EEG Acquisition and Preprocessing}

Continuous EEG was acquired at $1024 \mathrm{~Hz}$ though a 128-channel Biosemi Active Two system referenced to the common mode sense/driven right leg ground (which functions as a feedback loop driving the average potential across the montage as close as possible to the amplifier zero). EEG epochs from $100 \mathrm{~ms}$ before to $500 \mathrm{~ms}$ after stimulus onset (i.e., 102 data-points before and 514 data-points after stimulus onset) were extracted and then averaged for each

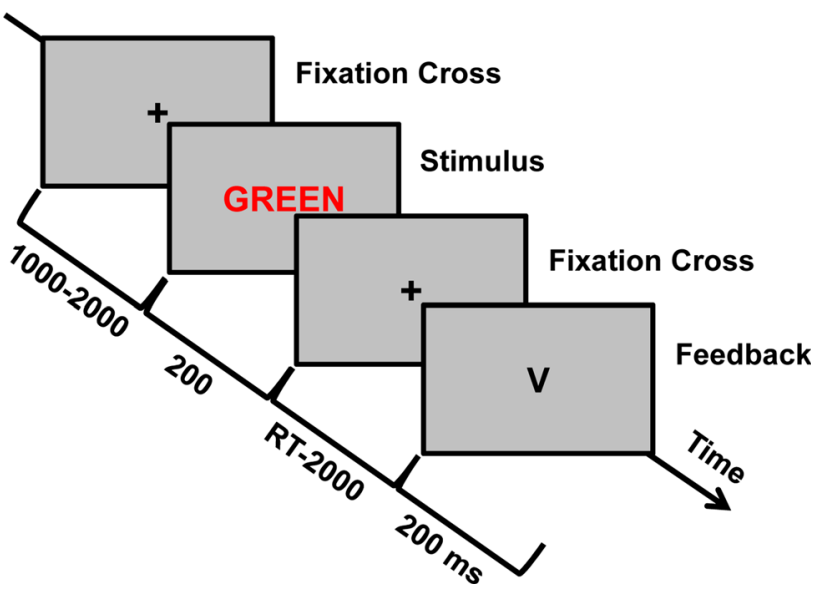

Fig. 1 Color Stroop task experimental paradigm. The four conditions (factor conflict strength: High and Low; factor congruency: Congruent and Incongruent color word and ink color) were presented randomly. Participants reported the color of the ink while ignoring the color named by the color word. After responding, subjects received a feedback on their accuracy. The timing of stimulus presentation, inter-stimulus interval, response window, and inter-trial interval are indicated (Color figure online)

participant. Trials with blinks, eye movements, or transient noise were rejected using a semi-automated $\pm 80 \mu \mathrm{V}$ criterion and visual inspection. This procedure generated four ERPs per participant, according to a $2 \times 2$ within-subject design with factors "conflict strength" (HC; LC) and "congruency" (congruent $(\mathrm{C})$; incongruent $(\mathrm{I})$ ) yielding to the experimental conditions $\mathrm{C} / \mathrm{HC}$; C/LC; I/HC; and I/LC. Prior to group averaging, artifact electrodes from each participant were interpolated (Perrin et al. 1987) and all electrodes were recalculated against average reference.

The average \pm SEM number of accepted artifact-free epochs was $223 \pm 4$ for the $\mathrm{C} / \mathrm{HC}, 224 \pm 4$ for the I/HC, $225 \pm 4$ for the C/LC, and $224 \pm 4$ for the I/LC conditions. A $2 \times 2$ repeated-measures ANOVA with factors of conflict strength and congruency (as performed for the behavioral and ERP analyses) on the number of accepted epochs revealed no main effect of congruency; no main effect of conflict strength and no significant interaction (all $p$ values $>0.17$ ). This ensured that our results did not simply follow from differences in the signal-to-noise ratio across experimental conditions.

\section{Event-Related Potentials Analyses}

Voltage Waveform Analyses A first level of analysis was performed by comparing the ERPs to the $\mathrm{C} / \mathrm{HC}$; I/HC; C/LC; and I/LC conditions using a $2 \times 2$ within-subject design with factors "conflict strength" (HC; LC) and "congruency" $(\mathrm{C} ; \mathrm{I})$ at each scalp electrode as a function of peri-stimulus time. The results of this ERP waveform analysis are presented as plot depicting the time frames 
showing a significant $(p<0.01)$ conflict strength $\times$ congruency interaction as a function of peri-stimulus time and electrodes. Correction was made for temporal auto-correlation through the application of a $>11$ contiguous datapoint temporal criterion for the persistence of differential effects (Guthrie and Buchwald 1991). The analysis was performed using the STEN toolbox. We provide ERP waveform from 6 exemplar electrodes and the result of traditional waveform analyses to help the reader assess the quality of the signal and contextualize our results with previous ERP literature on Stroop tasks. In addition, local analyses are likely more sensitive than global measure of the voltage field to small effects manifesting only over a limited number of electrodes and may thus provide information on whether small effect might have been missed by the global analyses. However, while these analyses give a visual impression of effects within the dataset, our conclusions are based on reference-independent global measures of the electric field at the scalp (Tzovara et al. 2012).

Reference-independent analyses of ERPs have several analytical and interpretational benefits over canonical ERP waveform (West and Alain 2000; Michel et al. 2004; Murray et al. 2008; Tzovara et al. 2012). These analyses circumvent interpretational issues attributable to the reference-dependent nature of ERPs and potential biases induced by a priori selection of a restricted set of electrodes or of time periods of interest. In addition, as detailed below, by contrast to local ERP analyses, the analyses of the global field power (GFP) and of the ERP topography enables to disentangle if the observed effects followed from change in responses gain and/or change in the configuration of the brain network across conditions, the central question of the present paper.

Global Electric Field Analyses Global electric field analyses were carried out using the RAGU and Cartool software (Koenig et al. 2011; Brunet et al. 2011). Modulations in the strength of the electric field at the scalp were assessed using the GFP (Lehmann and Skrandies 1980). GFP is calculated as the square root of the mean of the squared value recorded at each electrode (versus the average reference) and represents the spatial standard deviation of the electric field at the scalp. This calculation yields larger values for stronger electric fields. Differences in GFP waveform data as a function of time post-stimulus onset between the four conditions was analyzed using a $2 \times 2$ (conflict strength $\times$ congruency) within-subject design and randomization statistics: GFP at each time point was compared with an empirical distribution derived from a bootstrapping procedure (5000 permutations per data-point) based on randomly re-assigning each participant's data to either of the four conditions (see details in Koenig and Melie-Garcia 2010; Koenig et al. 2011). Only effects meeting or exceeding the $p<0.05$ criterion were considered as reliable. Correction was made for temporal auto-correlation through the application of a $>11$ contiguous data-point temporal criterion for the persistence of differential effects (Guthrie and Buchwald 1991).

Topographic modulations were identified using randomization statistics applied to global dissimilarity measures (DISS; Lehmann and Skrandies 1980). DISS is calculated as the root mean square of the difference between strengthnormalized vectors (here the instantaneous voltage potentials across the electrode montage). We analyzed DISS values as a function of time post-stimulus onset in a series of the same conflict strength $\times$ congruency within-subject design and randomization statistics as for the GFP analyses (Murray et al. 2008; Koenig and Melie-Garcia 2010; Koenig et al. 2011). DISS is independent of the reference electrode and is insensitive to pure amplitude modulations across conditions (i.e. DISS modulations are orthogonal to GFP modulations). As above, only effects meeting or exceeding the $p<0.05$ criterion were considered as reliable and temporal autocorrelation was corrected through the application of a $>11$ contiguous data-point temporal criterion (Guthrie and Buchwald 1991). This analysis is useful in terms of neurophysiologic interpretation because topographic changes necessarily follow from changes in the configuration of the brain's underlying active generators (Lehmann et al. 1987). When the different parts of a given brain networks change in strength differently across conditions, it results in a topographic modulation. In contrast, differences in GFP without concomitant difference in topography can be considered as following from all parts of the network modulating similarly in strength. Thus, DISS modulations can be understood as reflecting qualitative changes in the underlying brain networks whereas GFP modulations reflect quantitative changes. The results of the GFP and DISS analyses are displayed as the $p$ value (y-axis) as a function of time (x-axis), with periods of significant differences highlighted in red.

Electrical Source Estimations We estimated electric sources underlying scalp-recorded data using a distributed linear inverse solution based on a local autoregressive average (LAURA) regularization approach (Grave-de Peralta et al. 2004; also Michel et al. 2004 for a comparison of inverse solution methods). LAURA selects the source configuration that better mimics the biophysical behavior of electric fields (i.e. activity at one point depends on the activity at neighboring points according to electromagnetic laws). The solution space is based on a realistic head model and included 3,005 solution points homogeneously distributed within the grey matter of the average brain of the Montreal Neurological Institute (courtesy of R. Grave-de Peralta Menendez and S. Gonzalez Andino, University Hospital of Geneva, Geneva, Switzerland). Intracranial sources were estimated for each 
participant and condition from the ERP previously averaged over the period of interest defined by the topographic and/or GFP analyses. Source estimations were then statistically compared at each node level between conditions using the same conflict strength $\times$ congruency within-subject ANOVA as for the behavioral and electric field analyses. Only nodes with $p$ values $<0.01$ and clusters of at least 15 contiguous nodes were considered significant. This spatial criterion was determined using the AlphaSim program (http:// afni.nimh.nih.gov/afni/doc/manual/AlphaSim).

\section{Results}

\section{Proficiency}

The Oxford University Placement Test proficiency scores for the second language (French) ranged between 68 and $80 \%$ (mean $\pm \mathrm{SEM}=75 \% \pm 1.3$ ), which corresponds to medium proficiency. The BNT scores in L2 ranged between 39 and $56 \%(45.8 \% \pm 1.4)$ confirming that participants were of medium proficiency. The BNT scores in L1 ranged between 94 and $100 \%(98.8 \% \pm 0.7)$.

\section{Behavior}

Mean ( \pm SEM) response time was $629 \pm 25 \mathrm{~ms}$ for the $\mathrm{C} / \mathrm{HC}$ condition; $686 \pm 28 \mathrm{~ms}$ for the $\mathrm{I} / \mathrm{HC} ; 636 \pm 25 \mathrm{~ms}$ for the $\mathrm{C} / \mathrm{LC}$; and $678 \pm 27 \mathrm{~ms}$ for the I/LC (Fig. 2). Response times were submitted to a $2 \times 2$ repeated measure ANOVA with factors conflict strength (HC; LC) and congruency $(\mathrm{C} ; \mathrm{I})$. The factor conflict strength was manipulated by presenting the word in the participants' native (German, high conflict strength) versus second language (French; low conflict strength). There was a significant main effect of congruency (i.e. the Stroop effect; $\left.\mathrm{F}(1,11)=51,669 ; p<0.01 ; \mathrm{h}_{\mathrm{p}}^{2}=0.824\right)$ indicating that participants were generally slower in the incongruent than congruent condition, irrespective of the conflict strength. This difference was roughly parallel across L1 and L2. There was also a significant conflict strength $\times$ congruency interaction $\left(\mathrm{F}(1,11)=7,213 ; p<0.05, \mathrm{~h}_{\mathrm{p}}^{2}=0.396\right)$, driven by a larger Stroop effect in the high than in the low conflict condition. The main effect of conflict strength did not reach our $p<0.05$ significance criterion.

\section{EEG}

\section{ERP Wave-form Analyses}

Figure 3 displays the group-averaged ERPs to the four experimental conditions from six exemplar electrodes. Figure $4 \mathrm{a}$ shows the results of the analyses of ERP waveforms from the entire electrode montage as a function of time as well as the mean ERP topographies for each condition at the period of interest (420-445 ms). There was a main effect of conflict strength starting at ca. $250 \mathrm{~ms}$ and a main effect of congruency at $390 \mathrm{~ms}$ post-stimulus onset (Suppl. Fig. 1a). A widespread significant interaction between the two factors manifested at $\sim 390-460 \mathrm{~ms}$ following stimulus onset. However, as noted in the "Methods" section, reference-independent analyses of the global electric field were prioritized as they provide information on whether the effects stemmed from topographic and/or strength modulations and thus help formulating hypotheses on the neurophysiologic mechanisms underlying the observed ERP effects.

\section{Global Field Power}

The timeframe wise $2 \times 2$ analyses of GFP revealed a significant $(p<0.05, \mathrm{Ke}=11 \mathrm{TF})$ main effect of Congruency over the $377-500 \mathrm{~ms}$ post-stimulus onset period, as well as a main effect of Conflict Strength over the 90-115, 174-197 and 314-337 ms periods (Suppl. Fig. 1b). No significant interaction was found between these factors (Fig. 4c).

\section{Global Dissimilarity}

The timeframe wise $2 \times 2$ conflict strength $\times$ congruency analysis of global dissimilarity revealed a significant ( $p<0.05 ; \mathrm{Ke}=11 \mathrm{TF})$ main effect of congruency over the 72-107, 228-247 and the 385-500 ms post-stimulus onset period and a main effect of conflict strength at 163-205 and

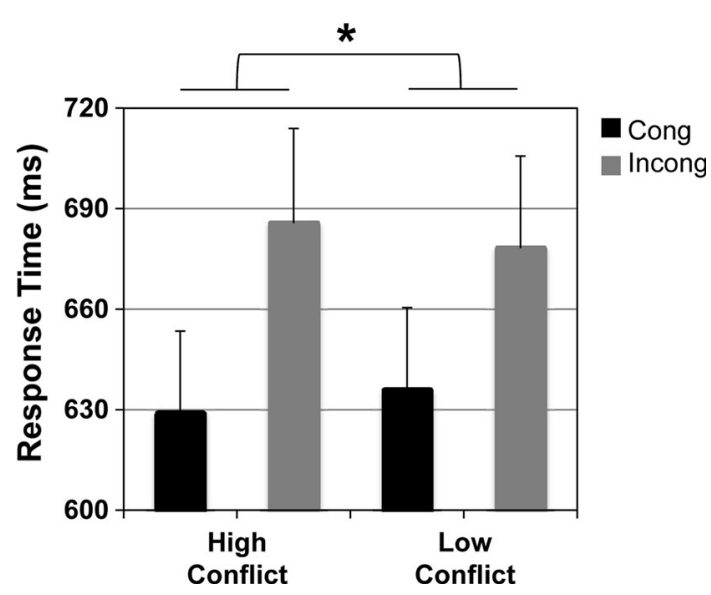

Fig. 2 Behavioral results. Group-averaged response time (in milliseconds) in reporting the color word ink color for each condition. There was a significant $(p<0.01)$ interaction between the factors conflict strength and congruency indicating a larger Stroop effect in the High Conflict than in the Low conflict condition (see "Results" for details) 
Fig. 3 Exemplar ERP waveforms. Group-averaged $(n=12)$ ERP waveforms from six exemplar electrodes for the four experimental conditions. The ERP in response to the congruent/high conflict $(\mathrm{C} / \mathrm{HC}$; red trace), incongruent/high conflict (I/HC; black trace), congruent/low conflict (C/LC; green trace) and incongruent/ low conflict (I/LC; blue trace) conditions are displayed in microvolts as a function of peristimulus time. The time periods with a significant conflict strength $\times$ congruency interaction are indicated in red (Color figure online)
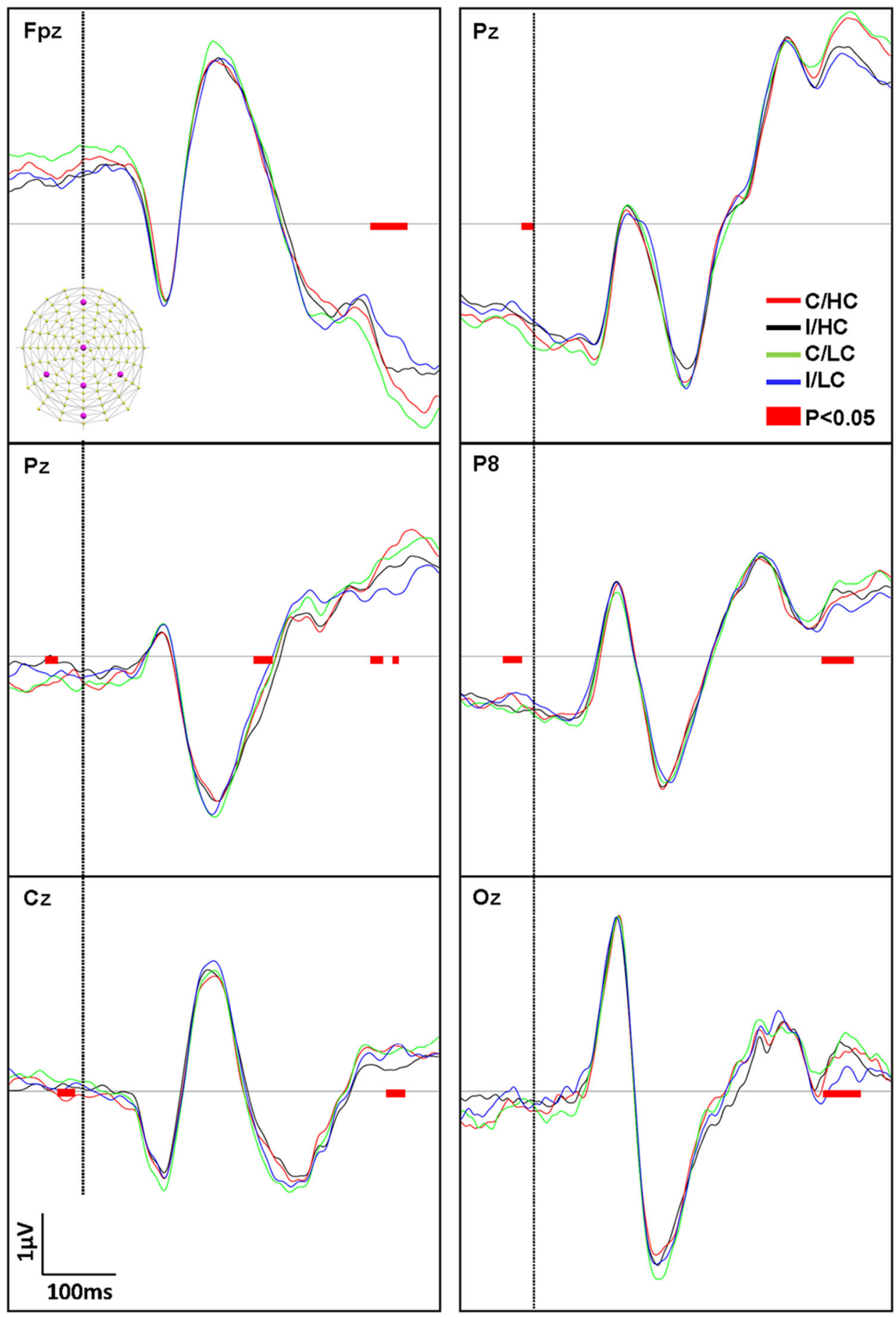

247-349 ms (Suppl. Fig. 1c). Importantly, an interaction between these factors manifested at 420-445 ms (Fig. 4b).

\section{Source Estimations}

Local autoregressive average distributed source estimations were calculated over the $420-445 \mathrm{~ms}$ post-stimulus period, i.e. when the topographic analyses showed a significant interaction between the factors conflict strength and congruency. To do so, ERPs for each participant and each experimental condition were first averaged separately across the above-mentioned time period of interest to generate one data-point per participant and experimental condition. Source estimations were then calculated. The Fig. 5a displays the grand mean source estimations for the four conditions over the $420-445 \mathrm{~ms}$ post-stimulus period. Then, the 
a Electrode-wise, time-wise ERP Waveforms analysis

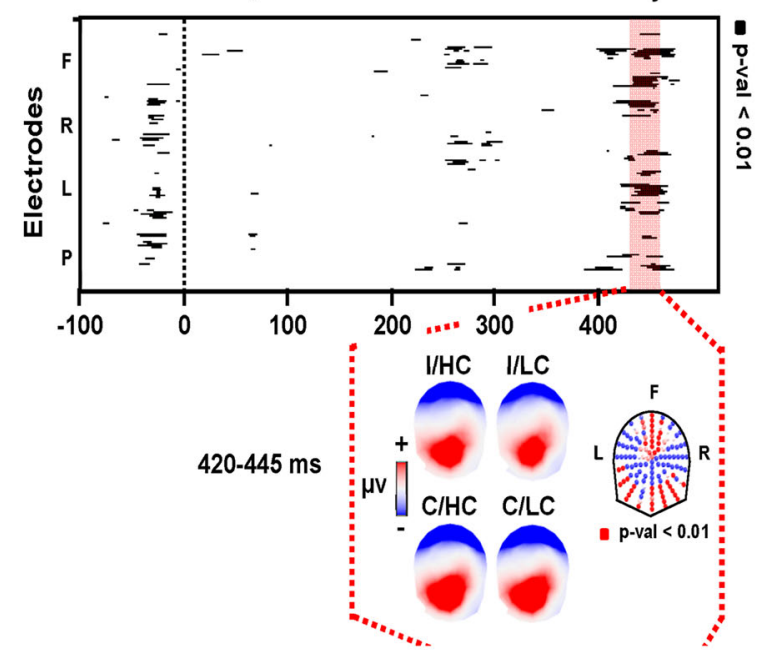

b Time-wise topographic analysis (Global Map Dissimilarity)

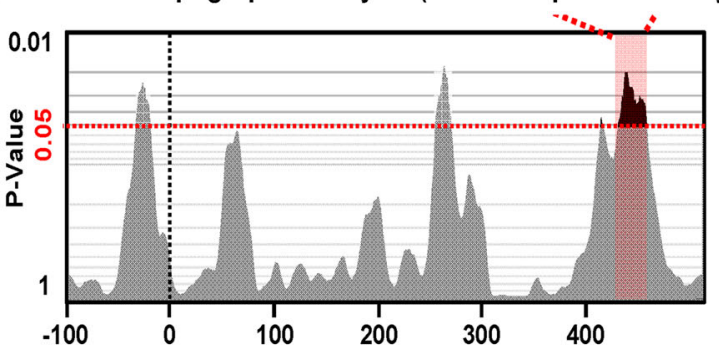

C Time-wise Global Field Power analysis
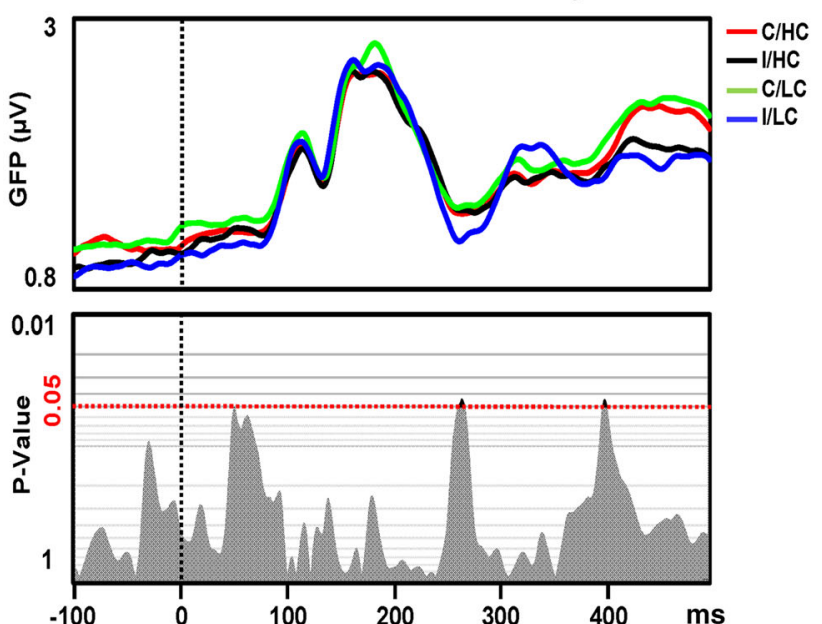

scalar value of each solution point (i.e. the current density) was submitted to the conflict strength $\times$ congruency ANOVA. There was a significant $(p<0.01)$ interaction within three distinct clusters: an occipital cluster centered around the left lingual gyrus and the left posterior cingulate gyrus; a frontal cluster centered on the ACC and extended bilaterally to the basal ganglia and insula; and a third cluster including the cingulate and paracentral lobule (Fig. 5b).
Fig. 4 Electrical neuroimaging results for the conflict strength $x$ congruency interaction. a Intensity plot, illustrating the significant $(p<0.01)$ statistical conflict strength $\times$ congruency interaction across the entire electrode montage. The $\mathrm{x}-, \mathrm{y}-$, and $\mathrm{z}$-axes illustrate respectively time, electrodes ( $F$ frontal, $R$ right, $L$ left, $P$ posterior) and $p$ value of the ANOVA (in black). The mean ERP topographies (in microvolt) over the period of interest (420-445 ms) for the four experimental conditions are represented. The red color represents the positive electric potentials and the blue the negative electric potentials. b Results of the global dissimilarity analysis for the interaction between factors conflict strength and congruency. The $p$ value of the interaction is plotted as a function of time; periods of significant topographic modulation $(p<0.05)$ are indicated in red. c Results of the Global Field Power (GFP) analysis for the interaction between factors conflict strength and congruency. GFP waveforms of the four experimental conditions (in microvolts, up panel) and the $p$ value of the interaction (bottom panel) are plotted as a function of time. There was no evidence for an interaction at the level of the GFP (Color figure online)

\section{Discussion}

This study examined whether situations of low and high conflict are processed by mechanism differing only in quantitative terms, or by qualitatively different networks processes. To do so, we investigated behavioral responses and electrical neuroimaging analyses to ERPs recorded during a color Stroop task in which we manipulated the level of conflict induced by task-irrelevant information, and thereby the demand for reactive inhibitory control. Behavioral results showed a significant Conflict Strength $\mathrm{x}$ Congruency interaction driven by a larger Stroop effect in the High Conflict (HC) than in the Low Conflict (LC) condition. Electrophysiologically, we observed a significant conflict strength $\times$ congruency interaction at the level of the ERPs topographies over the 420-445 ms poststimulus interval, a period corresponding to the $\mathrm{N} 450$ event-related potential (ERP) inhibition components typically observed in Stroop tasks (Liotti et al. 2000; West and Alain 2000). Because changes in topography necessarily follow from changes in the configuration of the underlying intracranial generators (e.g. Koenig and Gianotti 2009), our results indicate the engagement of distinct brain networks to resolve the Stroop interference in conditions of high versus low stimulus-driven conflict. There was no such interaction at the level of the GFP, further suggesting that mere changes in response gain did not account for the modulation in the Stroop effect by conflict strength. The conflict strength $\times$ congruency statistical analyses of electrical sources estimations performed over the period of topographic modulation revealed a significant interaction within a distributed cortico-subcortical frontal network including the ACC, basal ganglia, and middle frontal and occipital areas.

Our finding for a smaller behavioral Stroop effect in low than high conflict conditions replicates previous behavioral 
a Mean source estimations across conditions

$\mathrm{C} / \mathrm{HC}$
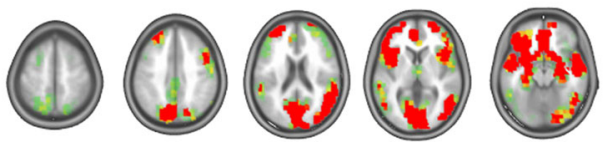

I/HC
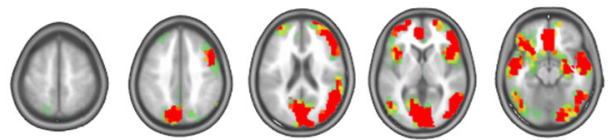

C/LC
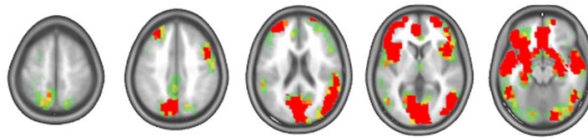

$8^{*} 10^{-4}$

$\Pi$

I/LC
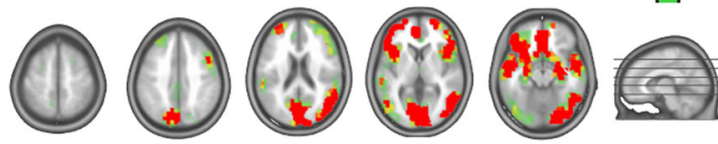

b Conflict Strength * Congruency Interaction
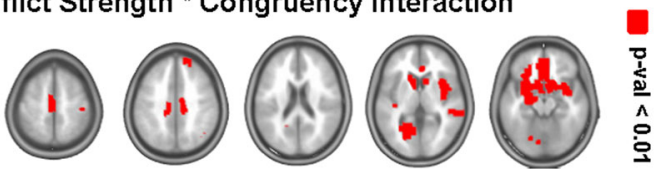

Fig. 5 LAURA electrical source estimations over the $420-445 \mathrm{~ms}$ period of significant topographic conflict strength $\times$ congruency interaction. a Grand mean source estimations of the four experimental conditions ( $C$ congruent, $I$ incongruent, $H C$ hight conflict, $L C$ low conflict). b Statistical analyses of the source estimations showing the significant conflict strength $\times$ congruency interaction $(p<0.01)$ over the $420-445$ post-stimulus period of topographic modulation

investigations of Stroop effects in which the strength of stimulus-induced conflict was manipulated (e.g. Mohamed Zied et al. 2004; Sumiya and Healy 2004, 2008; Braet et al. 2011; Youn 2011). These behavioral studies accounted for their pattern of results by positing that the brain mechanisms engaged to resolve high versus low conflict differed only quantitatively: the same inhibitory control mechanisms being engaged more strongly to cope with high than low conflict. Our electrophysiological results rather indicate that different brain networks were engaged depending on the strength of the conflict. If the neurophysiological mechanisms to control of high vs low conflicts differed only quantitatively, we would have observed an interaction at the level of the GFP without concomitant topographic modulations. Quantitative modulations in response amplitude have been for instance demonstrated in studies that manipulated task difficulty or attentional load (Hillyard and Anllo-Vento 1998; Luck et al. 2000). Repetition priming suppression effects have also been shown to result in GFP modulations without topographic changes, which was interpreted as a decrease in response strength of the same brain network following repeated exposure to the same stimuli (e.g. Murray et al. 2008). By contrast, we observed an interaction at the level of the topography but not in GFP, indicating qualitatively distinct brain networks for the inhibitory control of high versus low conflict (e.g. Murray et al. 2008; Koenig and Gianotti 2009). This pattern of results suggests that a modulation in the amplitude of the behavioral Stroop effect by variations in stimulus-induced conflict cannot be solely accounted for by an adjustment in the response strength of the same inhibitory control mechanism as previously assumed in psychophysical studies (Mohamed Zied et al. 2004; Sumiya and Healy 2004)

The conflict strength $\times$ congruency topographic interaction manifested around $400 \mathrm{~ms}$ post-stimulus onset. This latency corresponds to the time period when inhibitory processes engaged to detect and resolve Stroop interference are typically observed. Previous ERP studies on Stroop tasks indeed report differences between incongruent and congruent conditions over the $400-450 \mathrm{~ms}$ time period (so-called N450 ERP component; e.g. Liotti et al. 2000; Markela-Lerenc et al. 2004; Hanslmayr et al. 2008; Holmes and Pizzagalli 2008). Because the two conflict conditions were randomly intermixed within each block, variations in the anticipation of high or low conflict unlikely account for the conflict strength $\times$ congruency interaction. Proactive inhibition strategies have recently been highlighted for their critical role in determining how participants process conflicts (Aron 2011, for review). Increased activity in the striatum, supplementary motor areas, and the midbrain has been observed in situation with increased demand of inhibitory control (Zandbelt and Vink 2010; Zandbelt et al. 2012). Although no direct evidence demonstrate how proactive and reactive inhibition mechanisms interact, the brain network supporting these two mechanisms have been shown to largely overlap (Chikazoe et al. 2009; Jahfari et al. 2010; Zandbelt and Vink 2010; Swann et al. 2011; Zandbelt et al. 2012). Variation in proactive control induced by the anticipation of varying degrees of conflict might thus impact on reactive mechanisms. Mixing trials from the two languages probably equated proactive control at the block level, but it is not clear how the mixing controlled trial to trial variations in proactive control associated with incongruent stimuli.

The conflict strength $\times$ congruency interaction could be alternatively accounted for by the fact that distinct brain networks were engaged to control interfering information conveyed by the first (L1) and the second language (L2) because the two languages were supported by distinct representations. Speaking against this hypothesis, the main effect of conflict strength manifested around $250 \mathrm{~ms}$ poststimulus onset, i.e. $200 \mathrm{~ms}$ before the latency of the interaction. Together with previous literature, these findings suggest that along the temporal hierarchy of written word processing, the interaction was subsequent to the period when L1 and L2 words are differentially processed. In bilinguals with medium L2 proficiency and acquisition 
of L2 later than the seventh year as in the current study, differential brain responses to words in each language have typically been observed $150-350 \mathrm{~ms}$ post-stimulus onset within occipito-parietal areas and right hemispheric structures (Leonard et al. 2010; van Heuven and Dijkstra 2010; Howard et al. 1992). These effects have been related to the processing of lexico-semantic aspects of L1 and L2 and interpreted in terms of partially segregated processing pathways for each language over early stages of word processing (van Heuven and Dijkstra 2010). In line with our results, current views hold that the processing of each language in late, mid-proficient language learners involves distinct areas during the initial steps of lexico-semantic integration, but that verbal information subsequently converges to constitute common higher-level semantic representations independent of the original language (Abutalebi 2008). According to this view, inhibitory mechanisms engaged in the Stroop task most likely differed due to the difference in conflict strength from a common semantic representations rather than because L1 and L2 were supported by distinct representations (though it is not clear that ACC is activated by semantic conflict in the Stroop task; Chen et al. 2011). An additional argument supporting that the stimuli in L1 and L2 actually differed in term of conflict strength comes from studies reporting that similar conflict processing are engaged across the various conflict tasks (Botvinick et al. 2001; West 2003; West et al. 2005; though see Banich et al. 2000). For instance, West et al. (2005) showed that the N450 conflict components manifesting in Stroop, counting and digit location tasks was related to a single latent variable, suggesting that a common brain network supported conflict processing in these three tasks. If similar mechanisms indeed support the processing of conflict at the latency of our interaction, our results unlikely follow from differences in the nature of the conflict induced by the word in L1 and L2. Rather, our results suggest that it was indeed the variation in conflict strength that drove the interaction. In this regard, evidence for differences in conflict resolution mechanisms across tasks might thus be at least partly accounted for by differences in conflict strength (e.g. Banich et al. 2000).

The statistical analyses of electrical sources over the 420-445 ms time period of topographic modulation revealed that three brain regions exhibited a significant conflict strength $\times$ congruency interaction. A first cluster was centered on the ACC and extended bilaterally to the basal ganglia and insula. The ACC is typically involved in the stroop task and thought to support the detection and the resolution of the conflict between color word and ink color information (for review see Botvinick et al. 2001; Carter and van Veen 2007). Previous source modeling EEG study on Stroop task consistently pointed out the ACC and prefrontal regions as the main generator of the N450 components (Liotti et al. 2000; Markela-Lerenc et al. 2004; Hanslmayr et al. 2008; Badzakova-Trajkov et al. 2009; Bruchmann et al. 2010). The basal ganglia, notably including the caudate nucleus, have also been advanced to support the inhibition of the prepotent response schemes elicited by word reading during Stroop interference (Shadmehr and Holcomb 1999; Parsons et al. 2005; $\mathrm{Li}$ et al. 2008; Ali et al. 2010). Caudate nucleus and ACC have interestingly also been involved in language inhibition (Abutalebi 2008), but usually $200 \mathrm{~ms}$ before the interaction reported here (Khateb et al. 2007), suggesting different processes. However, we would note that although sparse evidence involved the basal ganglia in conflict tasks, to our knowledge no study pointed out this region as generating the N450 components. Although we applied a statistically robust parametric mapping analyses of source estimations, our results mostly revealed modulations within subcortical areas, whose activity is possibly less reliably detected by scalp-recorded EEG than superficial cortical activity. However, the source space used in the current study includes subcortical grey matter and distributed source estimations calculate the current density at all solution points. Recent evidence demonstrate that deep sources can be reliably estimated from scalp-recorded electrophysiological data (Lucka et al. 2012). Moreover, using the same inverse solution approach as in the current study, Michel et al. (2004) demonstrated that deep interictal middle temporal lobe epileptic activity can be accurately localized with our methods. These demonstrations however concern deep cortical rather than subcortical tissue as in the present results. Our result on the contribution of the basal ganglia to the N450 components should thus be interpreted with caution. In addition, the frontal cluster extended across functionally distinct cortical and subcortical regions (ACC, basal ganglia, insula); further studies based on neuroimaging methods with a higher spatial resolution than EEG are necessary to determine the precise role of these subregions in stroop task with varying conflict strength conditions.

Finally, the insular cortices have been involved in selective attention (Corbetta et al. 1991; Augustine 1996), which might be necessary in the Stroop task to prioritize the processing of the visual color over the word meaning information (Floden et al. 2011). The second cluster showing the conflict strength $\times$ congruency interaction was centered on the paracentral gyrus and the supplementary motor area. This region has been involved in controlling selective attention and might support the allocation of attentional resources to task-relevant information (e.g. Danielmeier et al. 2011), the inhibition of automatic or prepotent responses (e.g. Norman and Shallice 1986; Mayer et al. 2011), movement suppression (Schneider and Chein 2003) and decision making (Rogers et al. 1999; Volz 
et al. 2005). Accordingly, in our task the paracentral lobule could have been involved in responding to the ink color while inhibiting the competing information related to the meaning of the color word, as well as determining and producing the appropriate motor response. The third cluster included the left lingual gyrus and the left posterior cingulate. The former area has been involved in the processing of color and more specifically in attending to color information (Lueck et al. 1989; Corbetta et al. 1990; Harrison et al. 2005)

The question of segregated networks depending on conflict strength, demonstrated here in an intralanguage task, is also an important question in interlanguage paradigms (Guo et al. 2011). Cognitive inhibitory control has for example been proposed as a key mechanism of language control in bilinguals (Green 1998), for speech planning (Costa et al. 2000) or for lexical selection (Kroll et al. 2010). Compelling evidence suggest that cognitive control relies on the ACC and the caudate nucleus, and is modulated by language proficiency (Abutalebi et al. 2008; Abutalebi et al. 2013). This ability is critical in the selection of the less proficient language since it necessitate inhibiting the more proficient language. Supporting this hypothesis and our data for segregated networks for high versus low conflict strength, the selection of the less proficient language has been associated with an increase of left caudate nucleus responses (Abutalebi et al. 2013). Further analyses of the brain activity during low and high proficiency language selection could help determining whether segregated networks also support interlanguage inhibition.

A limitation of the present study includes the relatively small sample size. Although we had only 12 participants, we think that our study is sufficiently powered because we replicate the well-established effect of congruency, both behaviorally and electrophysiologically. Moreover, the non-parametric, randomization statistics for the GMD and GFP analyses appropriately deal with data from relatively small samples. Finally, we found the significant interaction on two statistically independent analyses conducted in the sensor- and in the brain-space.

Another potential confound concerns the inclusion of two female participants in our otherwise male sample. There is little evidence that gender impacts performance on the Stroop task and the rare electrophysiological studies on the effect of gender suggest that it may impact earlier components than those showing the interaction in our study (e.g. Shen 2005). More germane, since we used a withinsubject design, each participant was compared to his/herself. Should gender have interacted with any of our factors, this would have added noise in the data and increased the probability of type 2 errors.

To conclude, we note that our result for distinct inhibitory control mechanisms in $\mathrm{HC}$ and $\mathrm{LC}$ conditions contrasts with traditional conceptions of central top-down executive processes exerting control on subordinate cognitive processes (e.g. Norman and Shallice 1986; Aron 2007). In this regard, our finding for a variation in control mechanisms depending on the strength of the conflict driven by external event calls for incorporating evidence for within-tasks stimulus-dependent mechanisms in executive control, in addition to the well documented diversity of executive processes themselves (Miyake et al. 2000).

Acknowledgments This work was supported by grants from the Swiss National Foundation for Science to LS (\#320030-143348) and to JMA (\#32-138497). Cartool software has been programmed by Denis Brunet, from the Functional Brain Mapping Laboratory, Geneva, Switzerland, and supported by the Center for Biomedical Imaging (CIBM) of Geneva and Lausanne. The STEN toolbox has been programmed by Jean-François Knebel, from the Functional Electrical Neuroimaging Laboratory, Lausanne, Switzerland, and is supported by the Center for Biomedical Imaging (CIBM) of Geneva and Lausanne. We thank David Magezi for his valuable comments on an early version of the manuscript.

\section{References}

Abrams RA, Meyer DE, Kornblum S (1990) Eye-hand coordination: oculomotor control in rapid aimed limb movements. J Exp Psychol Hum Percept Perform 16(2):248-267

Abutalebi J (2008) Neural aspects of second language representation and language control. Acta Psychol (Amst) 128(3):466-478. doi: 10.1016/j.actpsy.2008.03.014

Abutalebi J, Annoni JM, Zimine I, Pegna AJ, Seghier ML, Lee-Jahnke H, Lazeyras F, Cappa SF, Khateb A (2008) Language control and lexical competition in bilinguals: an event-related FMRI study. Cereb Cortex 18(7):1496-1505. doi:10.1093/cercor/bhm182

Abutalebi J, Della Rosa PA, Ding G, Weekes B, Costa A, Green DW (2013) Language proficiency modulates the engagement of cognitive control areas in multilinguals. Cortex 49(3):905-911. doi:10.1016/j.cortex.2012.08.018

Ali N, Green DW, Kherif F, Devlin JT, Price CJ (2010) The role of the left head of caudate in suppressing irrelevant words. J Cogn Neurosci 22(10):2369-2386. doi:10.1162/jocn.2009.21352

Aron AR (2007) The neural basis of inhibition in cognitive control. Neuroscientist 13(3):214-228. doi:10.1177/1073858407299288

Aron AR (2011) From reactive to proactive and selective control: developing a richer model for stopping inappropriate responses. Biol Psychiatry 69(12):e55-e68. doi:10.1016/j.biopsych.2010. 07.024

Augustine JR (1996) Circuitry and functional aspects of the insular lobe in primates including humans. Brain Res Brain Res Rev 22(3):229-244

Badzakova-Trajkov G, Barnett KJ, Waldie KE, Kirk IJ (2009) An ERP investigation of the Stroop task: the role of the cingulate in attentional allocation and conflict resolution. Brain Res 1253:139-148. doi:10.1016/j.brainres.2008.11.069

Banich MT, Milham MP, Atchley R, Cohen NJ, Webb A, Wszalek T, Kramer AF, Liang ZP, Wright A, Shenker J, Magin R (2000) fMri studies of Stroop tasks reveal unique roles of anterior and posterior brain systems in attentional selection. J Cogn Neurosci 12(6):988-1000 
Botvinick MM, Braver TS, Barch DM, Carter CS, Cohen JD (2001) Conflict monitoring and cognitive control. Psychol Rev 108(3): 624-652

Braet W, Noppe N, Wagemans J, de Op Beeck H (2011) Increased Stroop interference with better second-language reading skill. Q J Exp Psychol (Hove) 64(3):596-607. doi:10.1080/1747 0218.2010.513735

Braver TS, Paxton JL, Locke HS, Barch DM (2009) Flexible neural mechanisms of cognitive control within human prefrontal cortex. Proc Natl Acad Sci USA 106(18):7351-7356. doi:10.1073/ pnas.0808187106

Britz J, Michel CM (2010) Errors can be related to pre-stimulus differences in ERP topography and their concomitant sources. Neuroimage 49(3):2774-2782. doi:10.1016/j.neuroimage.2009. 10.033

Bruchmann M, Herper K, Konrad C, Pantev C, Huster RJ (2010) Individualized EEG source reconstruction of Stroop interference with masked color words. Neuroimage 49(2):1800-1809. doi: 10.1016/j.neuroimage.2009.09.032

Brunet D, Murray M, Michel C (2011) Spatiotemporal analysis of multichannel EEG: cartool. Comput Intell Neurosci. doi: $10.1155 / 2011 / 813870$

Carter CS, van Veen V (2007) Anterior cingulate cortex and conflict detection: an update of theory and data. Cogn Affect Behav Neurosci 7(4):367-379

Chen A, Bailey K, Tiernan BN, West R (2011) Neural correlates of stimulus and response interference in a 2-1 mapping Stroop task. Int J Psychophysiol 80(2):129-138. doi:10.1016/j.ijpsycho. 2011.02.012

Chikazoe J, Jimura K, Hirose S, Yamashita K, Miyashita Y, Konishi S (2009) Preparation to inhibit a response complements response inhibition during performance of a stop-signal task. J Neurosci 29(50):15870-15877. doi:10.1523/JNEUROSCI.3645-09.2009

Corbetta M, Shulman GL (2002) Control of goal-directed and stimulus-driven attention in the brain. Nat Rev Neurosci 3(3): 201-215. doi: $10.1038 /$ nrn755

Corbetta M, Miezin FM, Dobmeyer S, Shulman GL, Petersen SE (1990) Attentional modulation of neural processing of shape, color, and velocity in humans. Science 248(4962):1556-1559

Corbetta M, Miezin FM, Dobmeyer S, Shulman GL, Petersen SE (1991) Selective and divided attention during visual discriminations of shape, color, and speed: functional anatomy by positron emission tomography. J Neurosci 11(8):2383-2402

Costa A, Caramazza A, Sebastian-Galles N (2000) The cognate facilitation effect: implications for models of lexical access. J Exp Psychol Learn Mem Cogn 26(5):1283-1296

Danielmeier C, Eichele T, Forstmann BU, Tittgemeyer M, Ullsperger M (2011) Posterior medial frontal cortex activity predicts posterror adaptations in task-related visual and motor areas. J Neurosci 31(5):1780-1789. doi:10.1523/JNEUROSCI.4299-10.2011

Floden D, Vallesi A, Stuss DT (2011) Task context and frontal lobe activation in the Stroop task. J Cogn Neurosci 23(4):867-879. doi:10.1162/jocn.2010.21492

Grass SM, Lee J (1984) Working memory capacity, inhibitory control, and proficiency in a second language. In: Schmid S.M, Lowie W (ed) Modeling Bilingualism: from the structure to chaos. John Benjamins Publishing Company, Philadelphia, p 59-84

Grave-de Peralta R, Gonzalez-Andino S, Gomez-Gonzalez CM (2004) The biophysical foundations of the localisation of encephalogram generators in the brain. The application of a distribution-type model to the localisation of epileptic foci. Rev Neurol 39 (8):748-756

Green DW (1998) Mental control of the bilingual lexico-semantic system. Bilingualism Lang Cogn 1(02):67-81. doi:10.1017/S136 6728998000133
Guo T, Liu H, Misra M, Kroll JF (2011) Local and global inhibition in bilingual word production: fMRI evidence from Chinese-English bilinguals. Neuroimage 56(4):2300-2309. doi:10.1016/j.neuroimage. 2011.03.049

Guthrie D, Buchwald JS (1991) Significance testing of difference potentials. Psychophysiology 28(2):240-244

Hanslmayr S, Pastotter B, Bauml KH, Gruber S, Wimber M, Klimesch W (2008) The electrophysiological dynamics of interference during the Stroop task. J Cogn Neurosci 20(2): 215-225. doi:10.1162/jocn.2008.20020

Harrison BJ, Shaw M, Yucel M, Purcell R, Brewer WJ, Strother SC, Egan GF, Olver JS, Nathan PJ, Pantelis C (2005) Functional connectivity during Stroop task performance. Neuroimage 24(1): 181-191. doi:10.1016/j.neuroimage.2004.08.033

Hillyard SA, Anllo-Vento L (1998) Event-related brain potentials in the study of visual selective attention. Proc Natl Acad Sci USA 95(3):781-787

Holmes AJ, Pizzagalli DA (2008) Response conflict and frontocingulate dysfunction in unmedicated participants with major depression. Neuropsychologia 46(12):2904-2913. doi:10.1016/ j.neuropsychologia.2008.05.028

Howard D, Patterson K, Wise R, Brown WD, Friston K, Weiller C, Frackowiak R (1992) The cortical localization of the lexicons. Positron emission tomography evidence. Brain 115(Pt 6): 1769-1782

Jahfari S, Stinear CM, Claffey M, Verbruggen F, Aron AR (2010) Responding with restraint: what are the neurocognitive mechanisms? J Cogn Neurosci 22(7):1479-1492. doi:10.1162/jocn.2009. 21307

Kaplan E, Goodglass H, Weintraub S (1983) Boston naming test. Lea and Febiger, Philadelphia

Kastner S, Ungerleider LG (2000) Mechanisms of visual attention in the human cortex. Annu Rev Neurosci 23:315-341. doi: 10.1146/annurev.neuro.23.1.315

Khateb A, Michel CM, Pegna AJ, Landis T, Annoni JM (2000) New insights into the Stroop effect: a spatio-temporal analysis of electric brain activity. NeuroReport 11(9):1849-1855

Khateb A, Abutalebi J, Michel CM, Pegna AJ, Lee-Jahnke H, Annoni JM (2007) Language selection in bilinguals: a spatio-temporal analysis of electric brain activity. Int $\mathrm{J}$ Psychophysiol 65(3):201-213. doi:10.1016/j.ijpsycho.2007.04.008

Koenig T, Gianotti L (2009) Scalp field maps and their characterization. In: Michel CM (ed). Electrical Neuroimaging. pp 25-48

Koenig T, Melie-Garcia L (2010) A method to determine the presence of averaged event-related fields using randomization tests. Brain Topogr 23(3):233-242. doi:10.1007/s10548-010-0142-1

Koenig T, Kottlow M, Stein M, Melie-Garcia L (2011) Ragu: a free tool for the analysis of EEG and MEG event-related scalp field data using global randomization statistics. Comput Intell Neurosci 2011:938925. doi:10.1155/2011/938925

Kroll JF, van Hell JG, Tokowicz N, Green DW (2010) The revised hierarchical model: a critical review and assessment. Biling (Camb Engl) 13(3):373-381. doi:10.1017/S136672891000009X

Lee TM, Chan CC (2000) Stroop interference in Chinese and English, vol 22. John Benjamins Publishing Company, Amsterdam, pp. 465-71 doi: 10.1076/1380-3395(200008)22:4;1-0;FT465

Lehmann D, Skrandies W (1980) Reference-free identification of components of checkerboard-evoked multichannel potential fields. Electroencephalogr Clin Neurophysiol 48(6):609-621

Lehmann D, Ozaki H, Pal I (1987) EEG alpha map series: brain micro-states by space-oriented adaptive segmentation. Electroencephalogr Clin Neurophysiol 67(3):271-288

Leonard MK, Brown TT, Travis KE, Gharapetian L, Hagler DJ Jr, Dale AM, Elman JL, Halgren E (2010) Spatiotemporal dynamics of bilingual word processing. Neuroimage 49(4):3286-3294. doi:10.1016/j.neuroimage.2009.12.009 
Li CS, Yan P, Sinha R, Lee TW (2008) Subcortical processes of motor response inhibition during a stop signal task. Neuroimage 41(4):1352-1363. doi:10.1016/j.neuroimage.2008.04.023

Liotti M, Woldorff MG, Perez R, Mayberg HS (2000) An ERP study of the temporal course of the Stroop color-word interference effect. Neuropsychologia 38(5):701-711

Liu X, Banich MT, Jacobson BL, Tanabe JL (2004) Common and distinct neural substrates of attentional control in an integrated simon and spatial Stroop task as assessed by event-related fMRI. Neuroimage 22(3):1097-1106. doi:10.1016/j.neuroimage.2004. 02.033

Logan GD (1980) Attention and automaticity in Stroop and priming tasks: theory and data. Cogn Psychol 12(4):523-553

Luck SJ, Woodman GF, Vogel EK (2000) Event-related potential studies of attention. Trends Cogn Sci 4(11):432-440

Lucka F, Pursiainen S, Burger M, Wolters CH (2012) Hierarchical bayesian inference for the EEG inverse problem using realistic FE head models: depth localization and source separation for focal primary currents. Neuroimage 61(4):1364-1382. doi: 10.1016/j.neuroimage.2012.04.017

Lueck CJ, Zeki S, Friston KJ, Deiber MP, Cope P, Cunningham VJ, Lammertsma AA, Kennard C, Frackowiak RS (1989) The colour centre in the cerebral cortex of man. Nature 340(6232):386-389. doi: $10.1038 / 340386 \mathrm{a} 0$

MacLeod CM (1991) Half a century of research on the Stroop effect: an integrative review. Psychol Bull 109(2):163-203

MacLeod CM, Dunbar K (1988) Training and Stroop-like interference: evidence for a continuum of automaticity. J Exp Psychol Learn Mem Cogn 14(1):126-135

Markela-Lerenc J, Ille N, Kaiser S, Fiedler P, Mundt C, Weisbrod M (2004) Prefrontal-cingulate activation during executive control: Which comes first? Brain Res Cogn Brain Res 18(3):278-287

Mayer AR, Teshiba TM, Franco AR, Ling J, Shane MS, Stephen JM, Jung RE (2011) Modeling conflict and error in the medial frontal cortex. Hum Brain Mapp. doi:10.1002/hbm.21405

Mead LA, Mayer AR, Bobholz JA, Woodley SJ, Cunningham JM, Hammeke TA, Rao SM (2002) Neural basis of the Stroop interference task: response competition or selective attention? J Int Neuropsychol Soc 8(6):735-742

Michel CM, Murray MM, Lantz G, Gonzalez S, Spinelli L, de Grave Peralta R (2004) EEG source imaging. Clin Neurophysiol 115(10):2195-2222. doi:10.1016/j.clinph.2004.06.001

Miller EK, Cohen JD (2001) An integrative theory of prefrontal cortex function. Annu Rev Neurosci 24:167-202. doi:10.1146/ annurev.neuro.24.1.167

Miyake A, Friedman N, Emerson M, Witzki A, Howerter A, Wager T (2000) The unity and diversity of executive functions and their contributions to complex "frontal lobe" tasks: a latent variable analysis. Cogn Psychol 41:49-100

Mohamed Zied K, Phillipe A, Pinon K, Havet-Thomassin V, Aubin G, Roy A, Le Gall D (2004) Bilingualism and adult differences in inhibitory mechanisms: evidence from a bilingual Stroop task. Brain Cogn 54(3):254-256. doi:10.1016/j.bandc.2004.02.036

Morishima Y, Akaishi R, Yamada Y, Okuda J, Toma K, Sakai K (2009) Task-specific signal transmission from prefrontal cortex in visual selective attention. Nat Neurosci 12(1):85-91. doi: $10.1038 / \mathrm{nn} .2237$

Morishima Y, Okuda J, Sakai K (2010) Reactive mechanism of cognitive control system. Cereb Cortex 20(11):2675-2683. doi: 10.1093/cercor/bhq013

Murray MM, Brunet D, Michel CM (2008) Topographic ERP analyses: a step-by-step tutorial review. Brain Topogr 20(4): 249-264. doi:10.1007/s10548-008-0054-5

Nieuwenhuis S, Donner TH (2011) The visual attention network untangled. Nat Neurosci 14(5):542-543. doi:10.1038/nn.2812
Norman W, Shallice T (1986) Attention to action, vol 4 consciousness and self regulation: advances in research and theory. Plenum, New York, pp 1-18

Oldfield RC (1971) The assessment and analysis of handedness: the Edinburgh inventory. Neuropsychol 9(1):97-113

Parsons MW, Harrington DL, Rao SM (2005) Distinct neural systems underlie learning visuomotor and spatial representations of motor skills. Hum Brain Mapp 24(3):229-247. doi: 10.1002/hbm.20084

Perrin F, Pernier J, Bertrand O, Giard MH, Echallier JF (1987) Mapping of scalp potentials by surface spline interpolation. Electroencephalogr Clin Neurophysiol 66(1):75-81

Peterson BS, Kane MJ, Alexander GM, Lacadie C, Skudlarski P, Leung HC, May J, Gore JC (2002) An event-related functional MRI study comparing interference effects in the Simon and Stroop tasks. Brain Res Cogn Brain Res 13(3):427-440

Ridderinkhof KR, van der Molen MW (1997) Mental resources, processing speed, and inhibitory control: a developmental perspective. Biol Psychol 45(1-3):241-261

Rogers R, Owen A, Middleton H, Williams E, Pickard J, Sahakian B, Robbins T (1999) Choosing between small, likely rewards and large, unlikely rewards activates inferior and orbital prefrontal cortex. J Neurosci 19(20):9029-9038

Rosselli M, Ardila A, Santisi MN, Arecco Mdel R, Salvatierra J, Conde A, Lenis B (2002) Stroop effect in Spanish-English bilinguals. J Int Neuropsychol Soc 8(6):819-827

Schneider W, Chein J (2003) Controlled and automatic processing: behavior, theory, and biological mechanisms. Cogn Sci 27:525-559

Shadmehr R, Holcomb HH (1999) Inhibitory control of competing motor memories. Exp Brain Res 126(2):235-251

Shen X (2005) Sex differences in perceptual processing: performance on the color-kanji Stroop task of visual stimuli. Int J Neurosci 115(12):1631-1641. doi:10.1080/00207450590958484

Stroop (1935) Studies of interference in serial verbal reactions. J Exp Psychol 18:643-662

Sumiya H, Healy AF (2004) Phonology in the bilingual Stroop effect. Mem Cogn 32(5):752-758

Sumiya H, Healy AF (2008) The Stroop effect in English-Japanese bilinguals: the effect of phonological similarity. Exp Psychol 55(2):93-101

Swann N, Poizner H, Houser M, Gould S, Greenhouse I, Cai W, Strunk J, George J, Aron AR (2011) Deep brain stimulation of the subthalamic nucleus alters the cortical profile of response inhibition in the beta frequency band: a scalp EEG study in Parkinson's disease. J Neurosci 31(15):5721-5729. doi: 10.1523/JNEUROSCI.6135-10.2011

Tillman CM, Wiens S (2011) Behavioral and ERP indices of response conflict in Stroop and flanker tasks. Psychophysiology 48(10):1405-1411. doi:10.1111/j.1469-8986.2011.01203.x

Tzovara A, Murray MM, Plomp G, Herzog MH, Michel CM, De Lucia M (2011) Decoding stimulus-related information from single-trial EEG responses based on voltage topographies. Pattern Recognit Advance online publication (in press)

Tzovara A, Murray MM, Michel CM, De Lucia M (2012) A tutorial review of electrical neuroimaging from group-average to singletrial event-related potentials. Dev Neuropsychol 37(6):518-544. doi:10.1080/87565641.2011.636851

van Heuven WJ, Dijkstra T (2010) Language comprehension in the bilingual brain: fMRI and ERP support for psycholinguistic models. Brain Res Rev 64(1):104-122. doi:10.1016/j.brainres rev.2010.03.002

van Veen V, Carter CS (2005) Separating semantic conflict and response conflict in the Stroop task: a functional MRI study. Neuroimage 27(3):497-504. doi:10.1016/j.neuroimage.2005. 04.042 
Volz KG, Schubotz RI, von Cramon DY (2005) Variants of uncertainty in decision-making and their neural correlates. Brain Res Bull 67(5):403-412. doi:10.1016/j.brainresbull.2005.06.011

West R (2003) Neural correlates of cognitive control and conflict detection in the Stroop and digit-location tasks. Neuropsychologia 41(8):1122-1135

West R, Alain C (2000) Effects of task context and fluctuations of attention on neural activity supporting performance of the Stroop task. Brain Res 873(1):102-111

West R, Jakubek K, Wymbs N, Perry M, Moore K (2005) Neural correlates of conflict processing. Exp Brain Res 167(1):38-48. doi:10.1007/s00221-005-2366-y
Youn H (2011) Computational neural model of the bilingual Stroop effect: a fMRI study, part 2: Ubiquitous computing and multimedia applications. In: In Tai-hoon K, Adeli H, Robles RJ, Balitanos M, editors. Communications in Computer and Information Science.. vol Berlin (DE):Springer. pp pp 60-65

Zandbelt BB, Vink M (2010) On the role of the striatum in response inhibition. PLoS ONE 5(11):e13848. doi:10.1371/journal.pone. 0013848

Zandbelt BB, Bloemendaal M, Neggers SF, Kahn RS, Vink M (2012) Expectations and violations: delineating the neural network of proactive inhibitory control. Hum Brain Mapp. doi:10.1002/hbm. 22047 